Estimating The Reliability of the

Lawrence Livermore National Laboratory (LLNL) Flash X-ray (FXR) Machine

M. M. Ong, R. Kihara, J. M. Zentler, B. R. Kreitzer, W. J. DeHope

June 27, 2007

2007 IEEE Pulsed Power and Plasma Science Conference Albuquerque, NM, United States

June 17, 2007 through June 22, 2007 
This document was prepared as an account of work sponsored by an agency of the United States Government. Neither the United States Government nor the University of California nor any of their employees, makes any warranty, express or implied, or assumes any legal liability or responsibility for the accuracy, completeness, or usefulness of any information, apparatus, product, or process disclosed, or represents that its use would not infringe privately owned rights. Reference herein to any specific commercial product, process, or service by trade name, trademark, manufacturer, or otherwise, does not necessarily constitute or imply its endorsement, recommendation, or favoring by the United States Government or the University of California. The views and opinions of authors expressed herein do not necessarily state or reflect those of the United States Government or the University of California, and shall not be used for advertising or product endorsement purposes. 


\title{
ESTIMATING THE RELIABILITY OF LAWRENCE LIVERMORE NATIONAL LABORATORY (LLNL) FLASH X-RAY (FXR) MACHINE*
}

\author{
Mike M. Ong ${ }^{\xi}$, Ron Kihara, Jan M. Zentler, Blake R. Kreitzer and William J. DeHope \\ Lawrence Livermore National Lab, PO Box 808, Mail Stop L-153 \\ Livermore, CA, USA
}

\begin{abstract}
At Lawrence Livermore National Laboratory (LLNL), our flash X-ray accelerator (FXR) is used on multi-million dollar hydrodynamic experiments. Because of the importance of the radiographs, FXR must be ultrareliable. Flash linear accelerators that can generate a $3 \mathrm{kA}$ beam at $18 \mathrm{MeV}$ are very complex. They have thousands, if not millions, of critical components that could prevent the machine from performing correctly. For the last five years, we have quantified and are tracking component failures. From this data, we have determined that the reliability of the high-voltage gas-switches that initiate the pulses, which drive the accelerator cells, dominates the statistics. The failure mode is a single-switch pre-fire that reduces the energy of the beam and degrades the X-ray spot-size. The unfortunate result is a lower resolution radiograph.

FXR is a production machine that allows only a modest number of pulses for testing. Therefore, reliability switch testing that requires thousands of shots is performed on our test stand. Study of representative switches has produced pre-fire statistical information and probability distribution curves. This information is applied to FXR to develop test procedures and determine individual switch reliability using a minimal number of accelerator pulses.
\end{abstract}

\section{Introduction}

The LLNL Flash X-ray machine is used on very expensive and important hydrodynamic experiments. (See Figure 1.) Therefore, FXR must be ultra-reliable. FXR generates a short $60 \mathrm{~ns}, 3 \mathrm{kA}$ electron beam with energy of $17 \mathrm{MeV}$. The goal is to expose a radiograph with sufficient X-ray dose, 400 rads, to penetrate the test object. The resolution of image is driven by X-ray spotsize that is currently about $1.8 \mathrm{~mm}$ full-width at halfmaximum. Four years ago, an optimization project was started to improve both the performance and reliability of this 25 year old machine $[1,2]$. This paper will focus on the reliability aspects. The overall reliability goal is to maintain our record of less than one lost radiograph per thousand hydrodynamic experiments. Hence, it is desirable to know the reliability of the machine before each experiment. This paper describes a statistical approach for estimating and predicting the reliability of FXR.

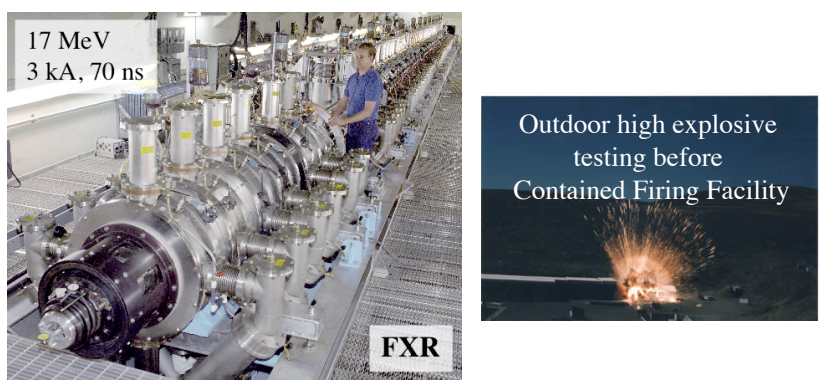

Figure 1. LLNL's Flash X-ray machine used on expensive and important hydrodynamic tests must be ultra-reliable.

High-dose and high-energy flash $\mathrm{x}$-ray machines are large and complex. FXR has hundreds of high-power pulsed power subsystems that generate and accelerator the beam and high-current supplies and magnets to transport and steer the beam to the X-ray converter. (See Figure 2.)
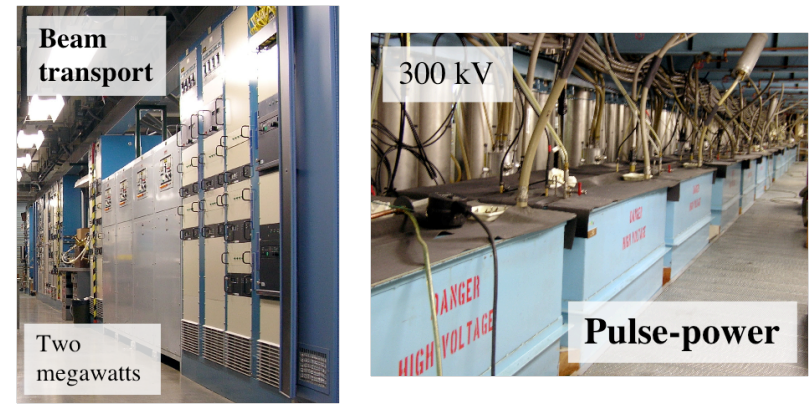

Figure 2. Large complex flash X-ray machines rely on hundreds of systems to function properly for a shot.

\footnotetext{
* This work was performed under the auspices of the U.S. Department of Energy by the University of California Lawrence Livermore National Laboratory under Contract No. W-7405-Eng-48.

§ email: ong1@1lnl.gov
} 
FXR has more than ten operations computers that set the timing system, control power supplies, and collect and display data on machine performance. The FXR subsystems include millions of electronic and mechanical components that could fail and prevent the machine from operating. In our experience, the reliabilities of the subsystems are not all equal. This project is taking a quantitative approach to identifying the problem areas and using statistics to predict the reliability of critical components and thus FXR. (See Figure 3.)

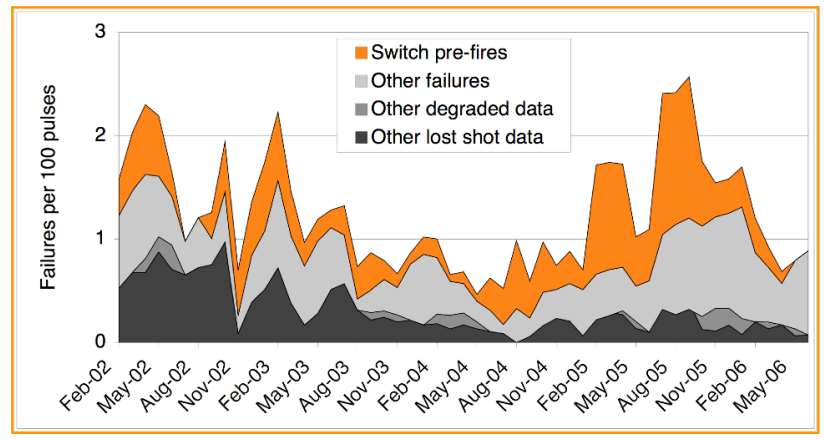

Figure 3. The performance of the high-voltage gas switches had a major impact on reliability statistics.

The reliability plot in Figure 3 shows different failure modes. The lost or degraded data are of the most concern, and they are attributed to high-voltage pre-fires and other mechanisms. At the end of 2006 a new SF6 gas system with very low water moisture content was developed to reduce the prefires. The switch reliability was much improved, and the pre-fire rate was too low to measure during normal operations. So, we set out to estimate the reliability of the gas switches.

\section{High-voltage Gas Switches}

The pulsed power system consists of a 5-stage Marx generator that is charged to $70 \mathrm{kV}$. (See upper portion of Figure 4.) When the Marx erects, a $300 \mathrm{kV}, 2 \mu \mathrm{s}$ pulse charges the Blumlein transmission line through a Veradyne Corporation gas switch. The bottom end of Blumlein is grounded by the switch that sends a $380 \mathrm{kV}$, $100 \mathrm{~ns}$ pulse to the accelerator cell. (See lower portion of Figure 4.) The SF6 gas switch was designed to operate consistently for many thousands of shots. Therefore, we believe it is possible to operate the switch for a large number of shots to gather statistical data.

All gas switches have some timing jitter after triggering, and the FXR Blumlein switches have only 1nsrms of jitter due to the strength of the trigger pulse. From the many measurements, the distribution of timing jitter appears to be Gaussian. Therefore, a switch closing 6 ns early should only occur once in ten million shots.

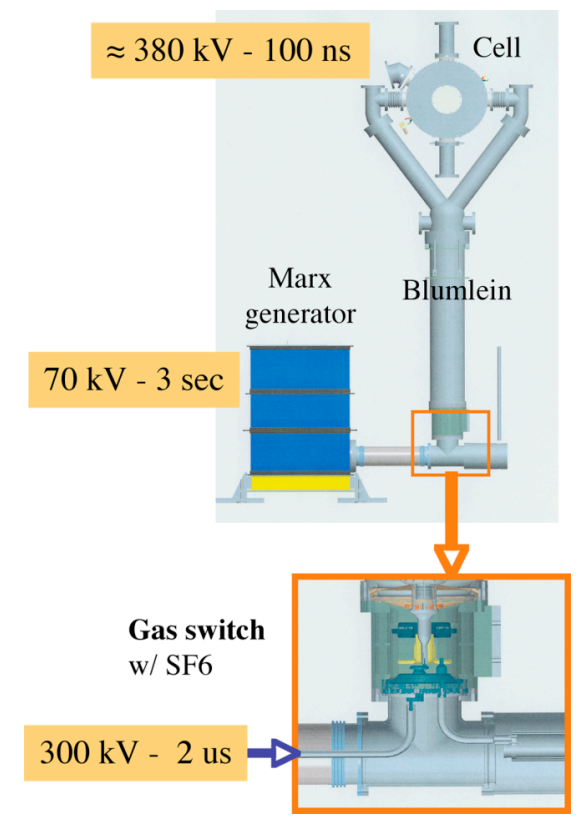

Figure 4. The FXR pulsed power system consists of Marx generators and Blumlein transmission lines.

For statistical purposes, we define pre-fire as a switch closure that occurs more than 6 ns before the trigger. In practice self-fires occur many hundreds of nanoseconds early. This reduces the energy of the beam. If the reduction occurs in the front of the accelerator, the electron beam will not be transported correctly to the Xray converter. Switch pre-fires are a major concern.

Three controllable parameters affect the reliability of the switch: Voltage, nominally $300 \mathrm{kV}$; SF6 gas pressure, 55 psig; and gas exchange volume, more than half per shot.

The FXR switch is normally triggered at the top of a $300 \mathrm{kV}$ pulse where there is little chance of a pre-fire. To determine the probability of a pre-fire, the switch was charged with an over-voltage pulsed with a peak of 450 $\mathrm{kV}$, and the trigger was disabled. (See Figure 5.)

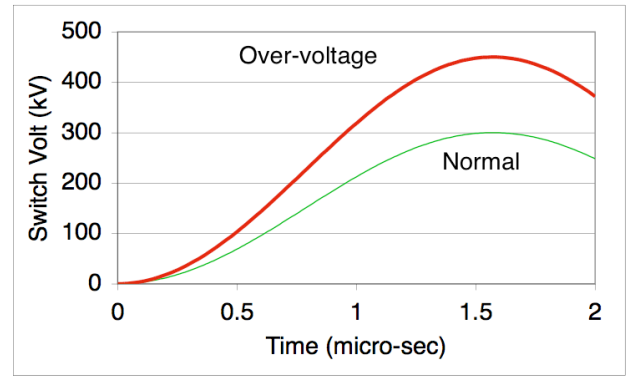

Figure 5. An over-voltage pulse is needed to generate pre-fire data.

Reducing the gas pressure will increase the pre-fire rate. (See Figure 6.) The hypothetical probability 
distribution and cumulative probability plots are based on a standard deviation of $7.5 \%$ that was determined from switch testing. The shift in voltage can be calculated with T. H. Martin's formula [3] that relates the gas density, $\rho$, the electric field in the gap, $E$, and time to closure, $\tau$.

$$
\left(\frac{\rho_{40 \text { psig }}}{\rho_{55 \text { psig }}}\right)^{2.44}=\frac{\left(\tau_{40 \text { psig }} E_{40 \text { psig }}^{3.44}\right)}{\left(\tau_{55 \text { psig }} E_{55 \text { psig }}^{3.44}\right)}[\text { Eq. 1] }
$$
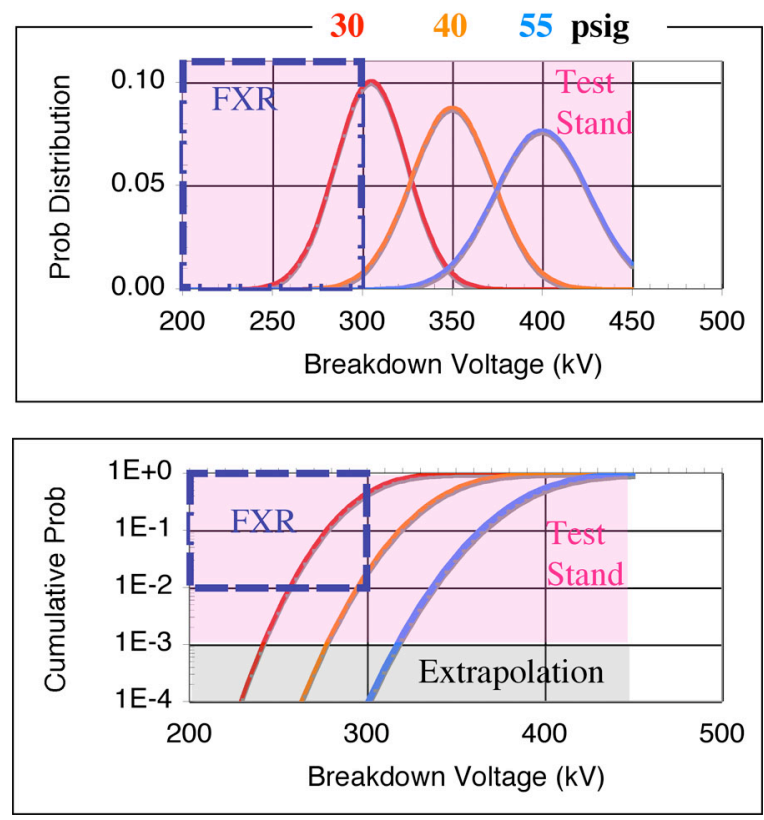

Figure 6. Hypothetical calculations show the effect of reducing gas pressure on pre-fire rates.

A million shots would be needed to accurately characterize the gas switches, and there is not enough time or funds to do this. With our Test Stand, exercising the switch thousands of times is acceptable, and it will provide data in the pink boxes in Figure 6. On FXR, 100 shots to characterize a particular switch once is acceptable as long as the pre-fire rate is reasonably low at a reduced pressure setting. This data will fall into the boxes labeled FXR in the plots. The extrapolation area is shown in the lower plot in Figure 6. Extrapolation to FXR reliability is only possible if the data points on the Test Stand and FXR overlap.

The ultimate goal is to check the reliability of each switch on FXR with only ten shots. The process for this will be explained in the next section.

\section{Test Result and Reliability Prediction}

On the Test Stand, the over-voltage waveform without trigger always caused the switch to always self-break with the nominal operating gas pressure of 55 psig. (See
Figure 7.) On FXR the switch is triggered at $300 \mathrm{kV}$, before any pre-fires in the plot.

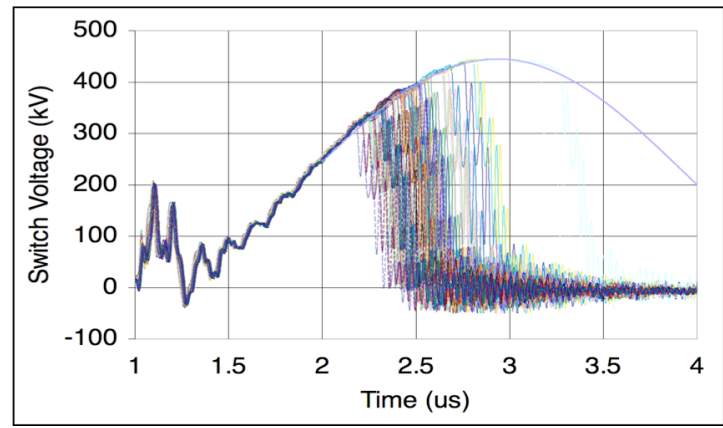

Figure 7. Over-voltage waveform caused the switch to always self-break.

Based on one thousand shots, the mean pre-fire voltage is $355 \mathrm{kV}$ as shown in the probability distribution plot in Figure 8. The cumulative probability is shown in the lower plot. Multiple Gaussian distributions appear to best describe the shape. However, for simplicity a single Gaussian will be used, and they are included in the plots as thin lines. The standard deviation is $6.7 \%$ of the mean value.
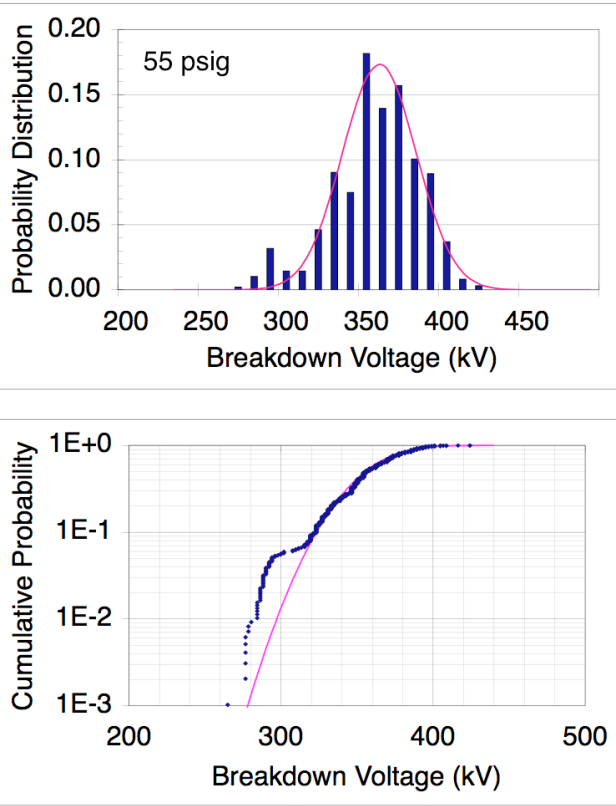

Figure 8. The probability distribution and cumulative probability are generated from the over-voltage test results.

When the gas pressure is reduced to 45 psig, the prefire voltage levels are lower. (See Figure 9.) In this example, two Gaussian distributions are clearly visible. The hypothesis is the switch is firing at two different electrode points. Using equation 1, the predicted mean voltage for the lower pressure is $305 \mathrm{kV}$. Based on all the 
data, the mean level is $300 \mathrm{kV}$, and the mean for the data on the left covered by the small Gaussian is $290 \mathrm{kV}$. The standard deviation is $6.7 \%$ for all the data, and $2.8 \%$ for the smaller Gaussian grouping.

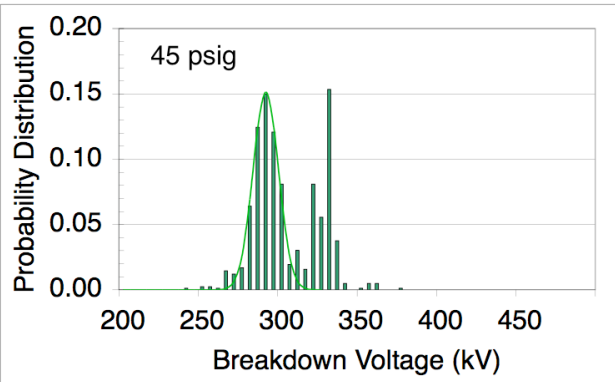

Figure 9. Test data at 45 psig shows two distinct Gaussian distributions.

Another parameter that affects the switch reliability is cleanliness of the SF6 gas. The gas volume exchange test results shown in Figure 10 were as expected. Each data point is generated from 200 shots. A full volume exchange improves the reliability. During the switch studies, a half volume exchange was deemed enough. For a hydrodynamic experiment, the gas is replaced multiple times.

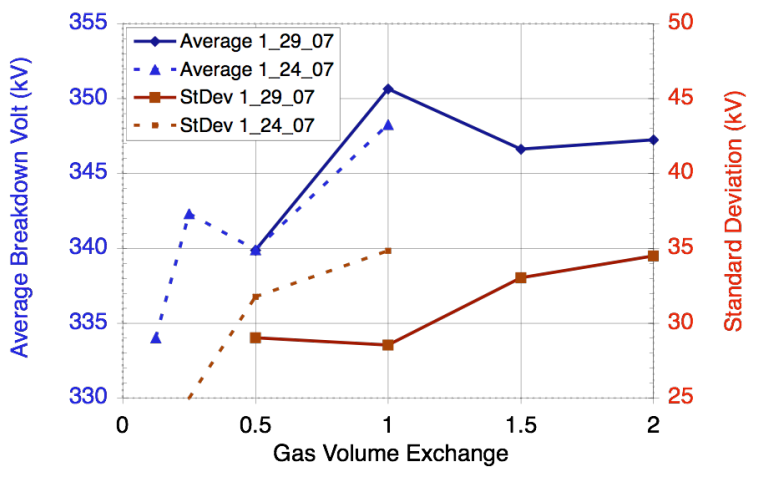

Figure 10. Gas volume exchange tests produced no surprises.

The goal of the reliability study is to quantify switch reliability on FXR with an expected pre-fire rate of less than $10^{-4}$. When the study has not been completed, a procedure has been developed to check switch reliability with ten shots. If a switch meets our reliability goal at 55 psig, then we ought to be able to select a lower pressure where the pre-fire rate is $50 \%$, hypothetically 30 psig.

Table 1 shows eleven possible outcomes from a switch check at the low gas pressure. The associated probability and the cumulative probability of such an outcome are also listed. If there are two or less pre-fires, the switch is considered reliable to a high level of confidence. For three to six pre-fires, the gas pressure could be raised or checked more often. If there are more than seven pre- fires, pressure must be raised and the switch repaired soon.

\begin{tabular}{|ccr|}
\hline Pre-fire's & P(pre-fire) & P-cumul \\
\hline 0 & 0.001 & $0 \%$ \\
1 & 0.010 & $1 \%$ \\
2 & 0.044 & $5 \%$ \\
3 & 0.117 & $17 \%$ \\
4 & 0.205 & $38 \%$ \\
5 & 0.246 & $62 \%$ \\
6 & 0.205 & $83 \%$ \\
7 & 0.117 & $95 \%$ \\
8 & 0.044 & $99 \%$ \\
9 & 0.010 & $100 \%$ \\
10 & 0.001 & $100 \%$ \\
\hline
\end{tabular}

Table 1. Low-pressure checks can identify reliable and problematic switches.

Two tasks still remain to be completed in this study. First, our test matrix with the over-voltage and normal voltage waveforms for various gas pressures must be filled in. Second, the effect of the two different waveforms on reliability must be explained.

\section{Summary}

It is possible to estimate and predict reliability of large complex machines like the LLNL FXR. It is possible to make FXR extremely reliable even if it includes highvoltage gas switches. Determining gas switch reliability will require a test stand. The resulting switch checking procedure will maximize the maintenance effort. FXR has already seen payoffs during a switch check.

\section{Acknowledgement}

We gratefully acknowledge the contributions of Keith Lewis who supported the FXR gas systems, Steve Beard for the mechanical upgrades on the Test Stand, Ed Koh for our computers, and Jim Dunlap for operating FXR. We also wish to thank Larry Wiley for providing the optimization funds.

\section{References}

[1] M. Ong, B. DeHope, K. Griffin, D. Goerz, R. Kihara, G. Vogtlin, J. M. Zentler, and R. Scarpetti, "Test Stand for Linear Induction Accelerator Optimization", IEEE International Pulsed Power Conference, June 2003.

[2] Mike M. Ong and George E. Vogtlin, "Flash X-ray (FXR) Accelerator Optimization Beam-induced Voltage Simulation and TDR Measurements", IEEE International Pulsed Power Conference, June 2005.

[3] T. H. Martin, "An Empirical Formula for Gas Switch Breakdown Delay", IEEE International Pulsed Power Conference, 1989. 